Mediterránea Ser. Biol. (1992/93), n. 14. Pág. 23-30

\title{
LA FAMILIA HAPLOZETIDAE GRANDJEAN, 1936 (ACARI, ORIBATIDA) EN LA PENÍNSULA IBÉRICA
}

\author{
por \\ J. GIL* Y L. S. SUBÍAS*
}

\section{RESUMEN}

Se hacen en este trabajo una serie de consideraciones sobre los géneros Haplozetes Willmann, 1935, Lauritzenia Hammer, 1958 e Incabates Hammer, 1961, quedando Haplozetes subdividido en dos subgéneros: $H$. (Haplozetes) y $H$. (Mixobates) n. subgen. y Laurizenia en otros dos subgéneros: L. (Lauritzenia) y $L$. (Incabates) n. stat. Además, se sacan todos los géneros, excepto Haplozetes, de la subfamilia Haplozetinae Grandjean 1936 "sensu" J. Balogh et P. Balogh, 1984 y se le sinonimiza la subfamilia Rostrozetinae J. Balogh et P. Balogh, 1984, creándose la subfamilia Lauritzeniinae n. subfam. para acoger a los géneros sacados de Haplozetinae. También se ofrecen redescripciones complementarias de dos especies de Haplozétidos ibéricos: Lauritzenia (Incabates) pallidus (Mihelčič, 1956) y Lauritzenia (Incabates) sinuatus (Pérez-Iñigo Jr., 1990).

PALABRAS ClAVE: Acaros, Oribátidos, Taxonomía, Haplozetidae, Península Ibérica.

\section{SUMMARY}

In this paper some considerations over genera Haplozetes Willmann, 1935, Lauritzenia Hammer, 1958 and Incabates Hammer, 1961 are made, remaining Haplozetes subdivided in two subgenera: $H$. (Haplozetes) and $H$. (Mixobates) n. subgen., and Lauritzenia also subdivided in two subgenera: $L$. (Lauritzenia) and $L$. (Incabates) n. stat. Moreover, all the genera of the subfamily Haplozetinae Grandjean, 1936 "sensu" J.Balogh el P. Balogh, 1984, except Haplozetes, are drawn out, and the subfamily Rostrozetinae J. Balogh et P. Balogh, 1984 is considered a synonym of the before mentioned; we have created the subfamily Lauritzeniinae n. subfam. to receive the genera that were before in the subfamily Haplozetinae.

We also give complementary redescriptions of two Iberian species of the family Haplozetidae Grandjean, 1936: Lauritzenia (Incabates) pallidus (Mihelčič, 1956) and Lauritzenia (Incabates) sinuatus (Pérez-Iñigo Jr., 1990).

KEY WORDS: Mites, Oribatids, Taxonomy, Haplozetidae, Iberian Peninsula.

* Cátedra de Entomología. Dpto. de Biología Animal I (Zoología). Facultad de Biología. Universidad Complutense. Madrid. 


\section{INTRODUCCIÓN}

Dados los reiterados problemas que se presentan en la clasificación de determinadas especies de la familia Haplozetidae Grandjean, 1936 encuadradas en el grupo de géneros que a continuación se comenta, se ha creido conveniente hacer una discusión de los mismos, a saber: Haplozetes Willmann, 1935, Lauritzenia Hammer, 1958 e Incabates Hammer, 1961. Para ello se van a utilizar básicamente los caracteres diagnósticos ya empleados en la diferenciación de géneros dentro de esta familia: el número de setas genitales y el número de uñas presentes en los tarsos.

\section{DISCUSIÓN TAXONÓMICA}

En primer lugar, y tomando el carácter "número de setas genitales" como diferenciador de géneros, tendríamos un género para las especies que presentan cinco pares de setas genitales, que sería Haplozetes, ya que la especie tipo de este género: Haplozetes vindobonensis Willmann, 1935, según redescripción de GRANDJEAN (1936) sobre ejemplares austriacos como los de la descripción original — corroborada por BECK (1964)—, presenta realmente cinco pares de setas genitales, en lugar de los cuatro que comenta WILLMANN (1935) en la descripción original; y otro género para las especies que presentan cuatro pares de seas genitales, que sería Lauritzenia ya que su especie tipo, Laurizenia longipluma Hammer, 1958, tiene dicho número. El género Incabates, creado por HAMMER (1961), que tiene a $I$. nudus como especie tipo, debe ser considerado muy próximo a Lauritzenia, ya que aparte de las restantes características comunes, también presenta cuatro pares de setas genitales.

En segundo lugar, y tomando como carácter diferenciador "el número de uñas en los tarsos", se pueden subdividir estos dos géneros, Haplozetes y Lauritzenia, en dos subgéneros cada uno (categoría que se da mientras no se conozca mejor el valor filogenético de este carácter). Dentro del género Haplozetes quedarían el subgénero $H$. (Haplozetes) para las especies que, como la especie tipo, tienen tarsos monodáctilos, y el subgénero $H$. (Mixobates) n. subgen. para las especies que tienen tarsos tridáctilos. Y dentro del género Lauritzenia quedarían el subgénero $L$. (Lauritzenia) para las especies que, como la especie tipo, presentan tarsos monodáctilos, y el subgénero L. (Incabates), estaría caracterizado por la posesión de cinco pares de setas genitales y tres uñas en sus tarsos, siendo la especie tipo de este taxón Protoribates (Protoribates) quadripilus Berlese, 1916.

Un carácter utilizado por BALOGH (1972) para separar Haplozetes y Lauritzenia, es la forma del sensilo, pero realmente éste sería similar en ambos, de tallo alargado y con el extremo dilatado en mayor o en menor medida; aparte de esto ambos presentan sáculos notogastrales y los pteromorfos móviles, al igual que Incabates.

A continuación, y una vez consultada toda la bibliografía con las descripciones originales de las especies hasta ahora pertenecientes a los tres 
géneros discutidos, se ofrece la redistribución de las especies en los nuevos taxones considerados.

\section{GÉNERO SUBGÉNERO ESPECIES}

Haplozetes (Haplozetes)

-5 pares de set. genit.

- monodactilia

Haplozetes (Mixobates)

-5 pares de set. genit.

- tridactilia.

Lauritzenia (Lauritzenia)

-4 pares de set. genit.

- monodactilia sp.t. Haplozetes vindobonensis Willmann, 1935

Haplozetes vindobonensis curtipilus Kunst, 1977.

Lauritzenia minuta Tseng. 1984.

Lauritzenia carneus Tseng. 1984.

sp.t. Protoribates (Protoribates) quadripilus Berlese, 1916

Haplozetes triungulatus beck, 1964.

Lauritzenia acutirostrum Hammer, 1968

Lauritzenia rotundirostrum Hammer, 1968

sp.t. Lauritzenia longipluma Hammer, 1958

Haplozetes minimocoma Beck, 1964.

Haplozetes cancellatus Beck, 1964

Haplozetes furtadoi Balogh et Mahunka, 1974

Haplozetes loongchiensis Tseng, 1984

sp.t. Incabates Hammer, 1961

Protoribates (Sheloribates) tenuifusus Berlese, 1916

Peloribates pallidus Mihelčič, 1956

Incabates angustus Hammer, 1967

Haplozetes elegans Kunst, 1977

Haplozetes sinuatus Pérez-Iñigo Jr., 1990.

Deben ser consideradas fuera del género Incabates - siguiendo el criterio de BALOGH (1972) y J. BALOGH et P. BALOGH (1984), que dan con pteromorfos móviles a la familia Haplozetidae -, todas las especies descritas en este género que tienen ptromorfos inmóviles, a saber: $I$. major Aoki, 1970, I. medius Hammer, 1971, I. pahabaeus Corpuz-Raros, 1980, I. striatus Corpuz-Raros, 1980 e I. aokii Choi, 1985. Y quedarían en posición indeterminada por falta de datos sobre el número de uñas o número de setas genitales - aunque dentro de Haplozetidae, pues presentan pteromorfos móviles-, las especies Incabates longisacculus Mahunka, 1984 y Haplozetes insignis Balogh et Mahunka, 1966, que estarían dentro de Lauritzenia, por presentar cuatro pares de setas genitales, pero de las que no se conoce el número de uñas de sus tarsos, y Haplozetes nudus (Hammer, 1958), que es una especie monodáctila, pero de la que se desconoce el número de setas genitales. 
La discusión hecha del género Haplozetes va a traer consigo la necesidad de hacer una serie de consideraciones sobre la subfamilia a que pertenece: Haplozetinae Grandjean, 1936 en el sentido que le dan J. BALOGH et P. BALOGH (op. cit.). Según dichos autores la subfamilia Haplozetinae estaría caracterizada por tener cuatro, o tres, pares de setas genitales, mientras que la subfamilia Rostrozetinae J. Balogh et P. Balogh, 1984 se diferenciaría por tener cinco pares de setas genitales. Al tener Haplozetes, su género tipo, cinco pares de setas genitales en base a su especie tipo, la subfamilia quedaría también así caracterizada. Por lo tanto la subfamilia Rostrozetinae pasaría a ser sinónima de Haplozetinae, mientras que los restantes géneros de la subfamilia Haplozetinae, con cuatro o tres pares de setas genitales, deben ser sacados de ésta creándose para ellos una nueva subfamilia, a la que se le ha dado el nombre de Lauritzeniinae n. subfam. Así la nueva subfamilia incluiría los géneros: Lauritzenia Hammer, 1958, Mancoribates Hammer, 1961, Magyaria Balogh, 1963, Magnobates Hammer, 1967 Conozetes Balogh et Mahunka, 1969, Flagellobates Mahunka, 1978 y Berlesiella Hammer, 1979.

\section{ESPECIES IBÉRICAS}

La primera especie de la familia Haplozetidae citada en España fue descrita originalmente por MIHELČIČ (1956), con el nombre de Peloribates pallidus sobre ejemplares recogidos en la Casa de Campo (Madrid). Posteriormente ha sido cambiada de género y citada como Scheloribates pallidus —PÉREZ-IÑIGO (1974) — y como Incabates pallidus - MÍNGUEZ (1981) - , siendo esta última ubicación la que se ha considerado en el presente trabajo.

La otra especie ibérica de Haplozétido es Haplozetes sinuatus descrita en el año 1990 por PÉREZ-IÑIGO Jr., sobre ejemplares recogidos en el Prepirineo de Huesca y que consideramos, por los caracteres que presenta ha de ser también incluida en $L$. (Incabates).

A continuación haremos una serie de observaciones sobre ambas especies.

Lauritzenia (Incabates) pallidus (Mihelčič, 1956) n. comb.

Peloribates pallidus MIHELČIČ, 1956

Scheloribates pallidus: PÉREZ-IÑIGO, 1974

Scheloribates pallidus: SUBIAS, 1977

Scheloribates pallidus: SUBIAS, 1980

Incabates pallidus: MÍNGUEZ, 1981

Incabates pallidus: KAHWASH et al., 1983

Incabates pallidus: SUBIAS et al., 1985

Incabates pallidus: ARILLO et al., 1988

Dimensiones: $285-315 \times 150-180 \mu \mathrm{m}$ (PÉREZ-IÑIGO, 1974)

$270-303 \times 145-162 \mu \mathrm{m}$ (RUIZ et al., 1986) 
En esta especie, los ejemplares que se han utilizado no presentan diferencias apreciables en visión dorsal, con los empleados en la redescripción de PÉREZ-IÑIGO (1974). Se representan por primera vez las visiones ventral (Figura 1a) y lateral (Figura 1b) como información complementaria sobre la especie.

Los ejemplares empleados en esta redescripción proceden de un muestreo realizado en 1975 por L-S. Subías en la Sierra de Cazorla (Sur de España).

Esta especie ha sido encontrada por todo el Centro y Sur de la Península Ibérica, y recientemente KARPPINEN et al. (1987) la han citado también del Caúcaso.

Lauritzenia (Incabates) sinuatus (Pérez-Iñigo Jr., 1990) n.comb.

Haplozetes sinuatus PÉREZ-IÑIGO Jr., 1990

Dimensiones: $276 \times 144 \mu$ m (PÉREZ-IÑIGO, 1990)

Los ejemplares empleados en esta redescripción, presentan algunas diferencias con respecto a los de la descripción original de esta especie. La primera diferencia es el tamaño, siendo estos ejemplares algo mayores $(279-343 \times 158-189 \mu \mathrm{m})$ que el descrito por PÉREZ-IÑIGO Jr. (1990). La segunda diferencia es la forma de los sáculos notogastrales, siendo piriformes en estos ejemplares (Figura 2a) mientras en el de la descripción original son tubiformes. La tercera diferencia se presenta en las setas del prodorso, que son total y finamente ciliadas (Figura 2a), mientras que en el original son lisas. La cuarta diferencia es el número de pares de setas epimerales, presentándose en estos ejemplares visibles todas las setas epimerales (Figura 2c) mientras que en el utilizado por su autor faltan, o al menos no los representa su autor, los pares $1 c, 3 c$ y $4 c$.

También se aporta una visión detallada del sensilo en posición lateral, observándose que es de extremo fusiforme-mazudo y finamente ciliado ( $\mathrm{Fi}$ gura $2 \mathrm{~b}$ ), además de una visión lateral del prodorso (Figura $2 \mathrm{~d}$ ), en la que se observan la seta exobotrídica $(e x)$ y el área porosa sublamelar $(A l)$.

Esta visión lateral es muy similar a la de $L$. (Incabates) pallidus, si bien en esta última especie el sensilo presenta un tallo más corto y el extremo más estrecho y fusiforme, siendo esta la diferencia fundamental entre las dos especies.

Los ejemplares empleados en esta redescripción son de un muestreo encabezado por L-S. Subías en el año 1983 en el Cabo de San Vicente (Sur de Portugal).

Hasta ahora sólo se conoce esta especie de la Península Ibérica. 

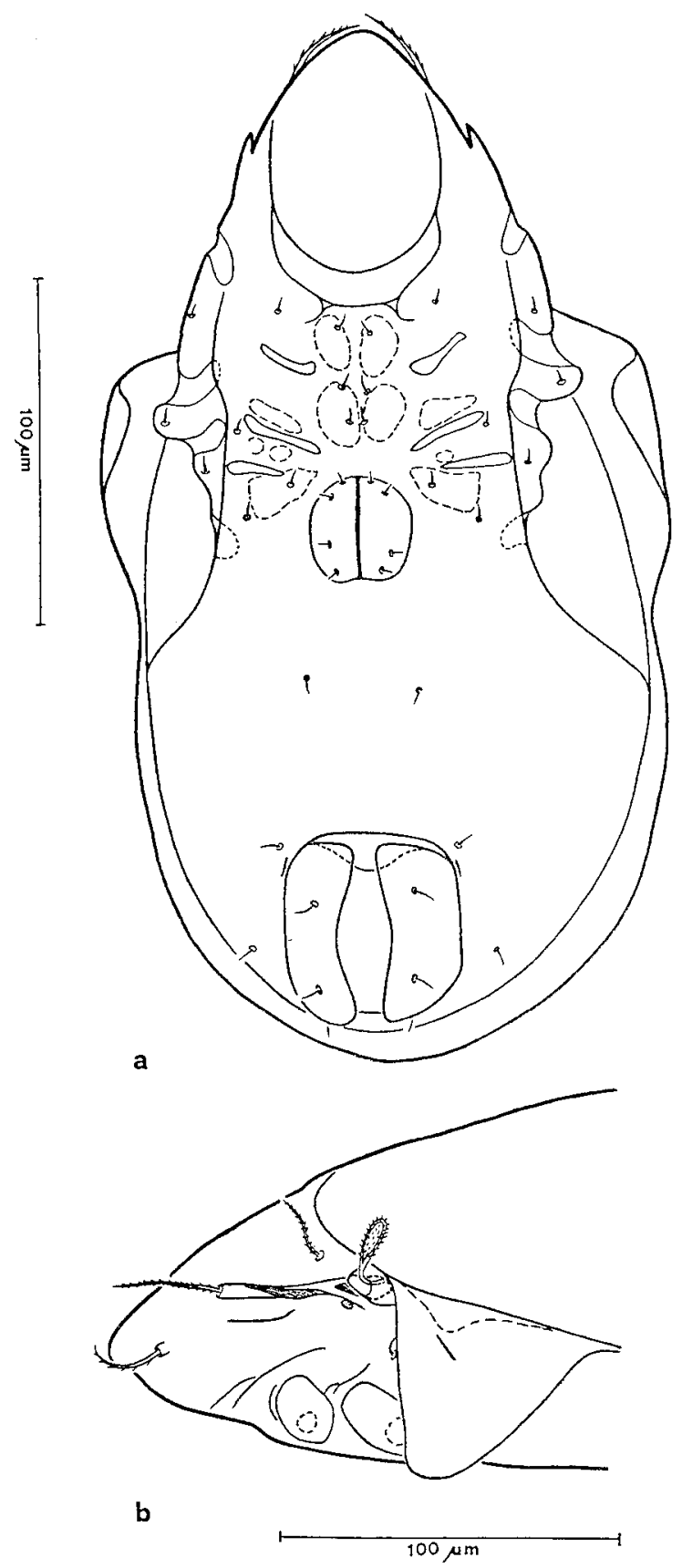

Figura 1.- Lauritzenia (Incabates) pallidus (Miheičič, 1956). a/ Visión ventral. b/ Visión lateral del prosorso.

Lauritzenia (Incabates) pallidus (Mihelčič, 1956). a/ Ventral view. b/ Lateral view of prodorsum. 


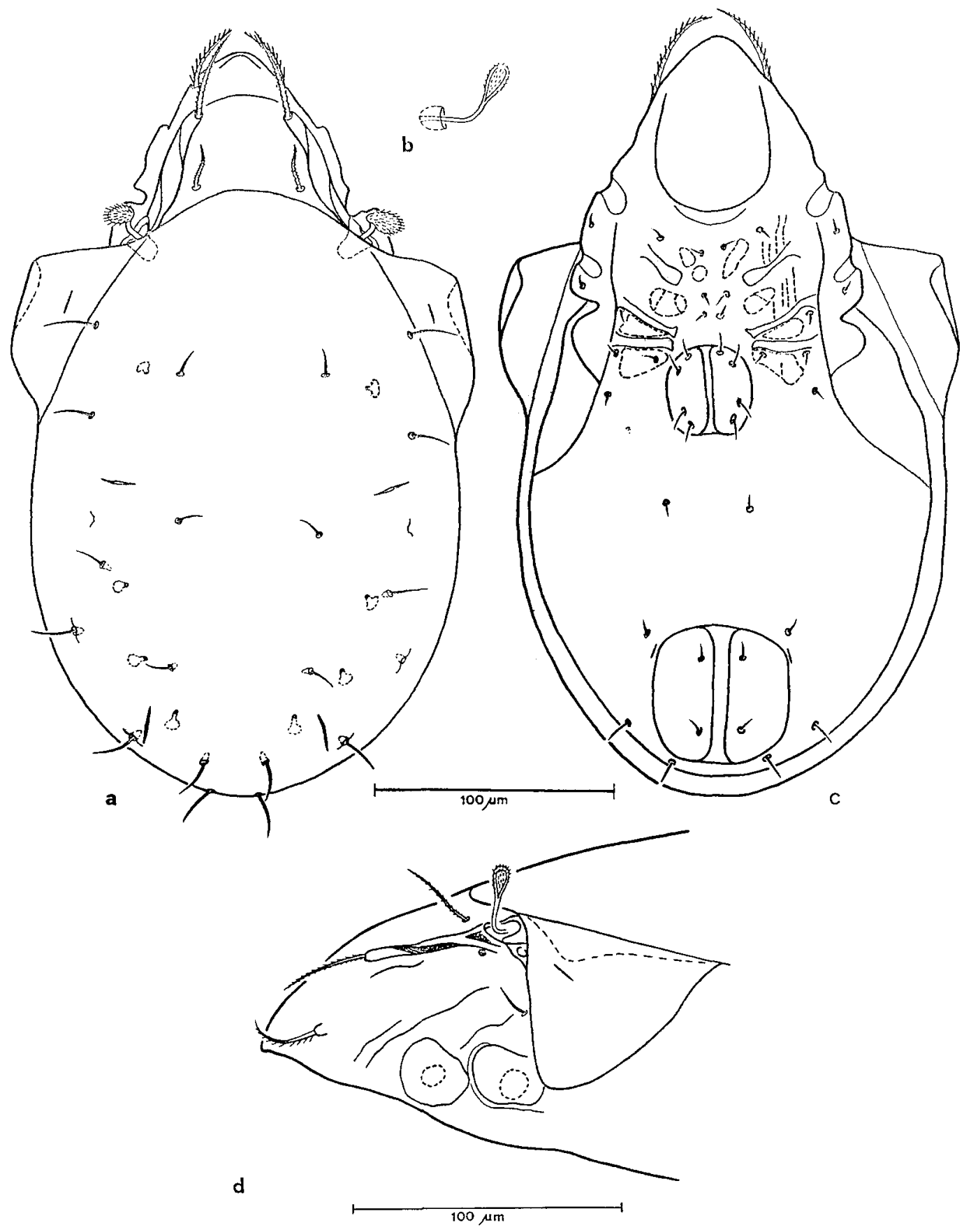

Figura 2.- Lauritzenia (Incabates) sinuatus (Pérez-Iñigo Jr. 1990). a/ Visión dorsal. b/ Sensilo en visión lateral. c/ Visión ventral. d/ Visión lateral del prodorso.

Lauritzenia (Incabates) sinuatus (Pérez-Iñigo Jr. 1990). a/ Dorsal view. b/ Lateral view of sensillus. $c /$ Ventral view. d/ Lateral view of prodorsum. 


\section{BIBLIOGRAFÍA}

ARILLO, A.; BORDEL, I. et SUBÍAS, L.S., 1988. Los Oribátidos (Acari, oribatida) de la Ciudad Universitaria de Madrid. Bol. R. Soc. Esp. Hist. Nat. (Biol.), 84 (1-2): 117-125.

BALOGH, J. 1972. The Oribatid genera of the world. Akadémiai Kiadó. Budapest. 188 pp.

BALOGH, J. et BALOGH, P. 1984. A review of the Oribatuloidea Thor, 1929 (Acari, Oribatei). Act. Zool. Hung., 30 (3-4): 257-313.

BALOGH, J. et MAHUNKA, S. 1966. The scientific results of the hungarian soil zoological expedition to the Brazzaville-Congo. 3. The Oribatid mites (Acari) of Brazzaville-Congo. I. Act. Zool. Acad. scien. Hung., 12 (1-2): 25-40.

BECK, L. 1964. Beiträge zur Kenntnis der neotrpischen oribatidenfauna. 4. Haplozetes und Peloribates (Arach., Acari). Senck. biol., 45: 161-183.

GRANDJEAN, F. 1936. Observations sur les Oribates (10 série). Bull. Mus. nat. Hist. natur. (2), 8: 246-253.

HAMMER, M. 1961. Investigations on the Oribatid fauna of the Andes Mountains. II. Perú, Biol. Skr. Dan. Vid. Selsk., 13 (1): 1-159.

KAHWASH, M.A., MÍNGUEZ, M. E. et SUBÍAS, L. S. 1983. Estudio taxocenótico de los Oribátidos (Acarida) de una zona agrícola de Toledo. Act. I. Congr. iber, Entom., II: 459-470.

KARPPINEN, E.; KRIVOLUTSKY, D. A.; TARBA, Z. M., SHTANCHAEVA, U. Ya. et GORDEEVA, E. W. 1987. List of Oribatid mites (Acarina, Oribatei) of northern palaearctic region. IV. Caucasus and Crimea. Ann. Ent. Fenn., 53: 119-137.

MIHELČIČ, F. 1956. Oribatiden Südeuropas III. Zool. Anz., 156: 9-29.

MíngUEZ, M.E. 1981. Estudio taxocenótico de los Oribátidos (Acarida, Oribatida) de El Pardo. Ed. Univ. Compl. Madrid. 281 pp.

PÉREZ-IÑIGO, C. 1974. Acaros Oribátidos de suelos de España peninsular e Islas Baleares (Acari, Oribatei). Parte V. Eos, 48: 367-475.

PÉREZ-INIGO, C. Jr. 1990. Acaros oribátidos (Acari, Oribatei) de la provincia de Huesca. I prepirineo. Eos, 65 .

RUIZ, E.; MÍNGUEZ, M. E. et SUBÍAS, L. S. 1986. Los Oribátidos (Acari, Orribatida) de los eriales de cultivo de una zona agrícola del sur de Madrid y el efecto borde. Act. VIII. Jorn. Asoc. esp. Ent.: 98-110.

SUBÍAS, L. S. 1977. Taxonomía y Ecología de los Oribátidos Saxícolas y Arborícolas de la Sierra de Guadarrama (Acarida, Oribatida). Trab. Cát. Artr. Fac. Biol. Univ. Compl. Madrid., n. 24. 374 pp.

SUBÍAS, L. S. 1980. Acaros Oribátidos de la Sierra de Cazorla (Acarida, Oribatida). En: Ed. M. G. de Viedma. Fauna de Cazorla. Invertebrados. ICONA. Monografía n. ${ }^{23}$ : 7-51.

SUBÍAS, L. S.; RUIZ, E. et MÍNGUEZ, M. E. 1985. Aportación al conocimiento de las comunidades de Oribátidos (Acari) del erial mediterráneo. Act. II. Congr. iber. Entom., I: $389-398$.

WILLMANN, C. 1935. IV Die Milben-fauna. Zool. Jahrb. Jena (Syst). 66: 331-344. 